\title{
Plastome phylogeny and early diversification of Brassicaceae
}

\author{
Xinyi Guo ${ }^{1}$, Jianquan Liu ${ }^{1 *} \mathbb{D}$, Guoqian Hao ${ }^{1,2}$, Lei Zhang ${ }^{1}$, Kangshan Mao ${ }^{1}$, Xiaojuan Wang ${ }^{1}$, Dan Zhang ${ }^{1}$, Tao Ma', \\ Quanjun Hu', Ihsan A. Al-Shehbaz ${ }^{3}$ and Marcus A. Koch ${ }^{4}$
}

\begin{abstract}
Background: The family Brassicaceae encompasses diverse species, many of which have high scientific and economic importance. Early diversifications and phylogenetic relationships between major lineages or clades remain unclear. Here we re-investigate Brassicaceae phylogeny with complete plastomes from 51 species representing all four lineages or 5 of 6 major clades (A, B, C, E and F) as identified in earlier studies.
\end{abstract}

Results: Bayesian and maximum likelihood phylogenetic analyses using a partitioned supermatrix of 77 protein coding genes resulted in nearly identical tree topologies exemplified by highly supported relationships between clades. All four lineages were well identified and interrelationships between them were resolved. The previously defined Clade $C$ was found to be paraphyletic (the genus Megadenia formed a separate lineage), while the remaining clades were monophyletic. Clade E (lineage III) was sister to clades B $+C$ rather than to all core Brassicaceae (clades $A+B+C$ or lineages $I+I I)$, as suggested by a previous transcriptome study. Molecular dating based on plastome phylogeny supported the origin of major lineages or clades between late Oligocene and early Miocene, and the following radiative diversification across the family took place within a short timescale. In addition, gene losses in the plastomes occurred multiple times during the evolutionary diversification of the family.

Conclusions: Plastome phylogeny illustrates the early diversification of cruciferous species. This phylogeny will facilitate our further understanding of evolution and adaptation of numerous species in the model family Brassicaceae.

Keywords: Plastome, Brassicaceae, Phylogenomics, Molecular dating, Gene loss

\section{Background}

The predominantly herbaceous family Brassicaceae (Cruciferae), which has some 3700 species, includes many vegetable crops in the genera Brassica and Raphanus, sources of spices (Eutrema and Armoracia) and vegetable oils (Brassica), ornamentals (Arabis, Hesperis, Lobularia, and Matthiola), and model species in experimental biology (e.g., Arabidopsis thaliana). A robust phylogeny is crucial for diverse comparative studies. However, resolving the deep phylogeny of the family has been particularly challenging because its early evolution was extremely rapid [1-5], accompanied with ancient gene flow [6], polyploidization [7-9], and origin of novel traits [10]. Prior phylogenetic studies, which involved 325 genera and 51 tribes using sequence variations of a few chloroplast

\footnotetext{
*Correspondence: liujq@nwipb.cas.cn

'MOE Key Laboratory of Bio-Resources and Eco-Environment, College of Life Sciences, Sichuan University, 610065 Chengdu, People's Republic of China
} Full list of author information is available at the end of the article
DNAs or ITS, identified four major lineages, with the basal lineage (tribe Aethionemeae) sister to the remaining three lineages (I, II, and III, i.e., core Brassicaceae) [2-5, 11-16]. The relationships between lineages within core Brassicaceae remained unsolved or inconsistent in those studies. Most recently, six clades were proposed based on phylogenetic analyses of low-copy nuclear genes retrieved from transcriptomes of 55 species [17]. The study further divided lineage II into three clades $(B, C, D)$, but the remaining three clades were similar to the previously recognized three lineages (basal lineage, and lineages I and III). The clade $\mathrm{E}$ (lineage III) was sister to the remaining core Brassicaceae species (clades A $+\mathrm{B}+\mathrm{C}$ or lineages $\mathrm{I}+$ II), but the relationship within the core were unsolved in the previous study [5]. Phylogenetic conflicts between different datasets, especially between nuclear and cytoplasmic genomes in plants, were found $[18,19]$, possibly suggesting complex evolutionary history. 
The chloroplast genomes (plastomes), with extremely more informative sites for phylogenetic analyses than only a few DNA fragments, have proven to be highly effective in resolving disputed interrelationships in numerous plant groups [20-22]. Plastomes vary in size between 75 and $250 \mathrm{~kb}$, have numerous copies in a given cell, inherited maternally in most plants, and have conserved gene content and order [23, 24]. The plastome is characterized by two usually large inverted repeat regions (IRa and IRb) separated by two single-copy regions referred to as the large single-copy region (LSC) and small single-copy region (SSC) that vary in length. Occasional structural changes, such as gene or intron losses, inversions, and rearrangements, were revealed by comparative genomic studies between groups. For examples, numerous plastome genes were lost multiple times in parasitic, nonphotosynthetic plants such as species of Cuscuta [25, 26], Epifagus [27], and Rhizanthella [28]. In photosynthetic species, the loss of chloroplast genes rarely occurs and only when the nuclear and/or mitochondrial genomes contain another functional copy or acquired one from the plastome through gene transfer [29]. Such rare cases were found in the rpl22 gene in Fagaceae and Passifloraceae [30], the rpl32 gene in Populus [31], and the infA gene in Brassicaceae [32]. Therefore, the relative stability of plastomes in plants provide highly orthologous alignments of large genome data that are valuable for phylogenetic analyses and calibrated divergence estimation [33-35].

The first plastome phylogeny of Brassicaceae have recently been presented aiming to provide a reliable temporal evolutionary framework within the entire clade of Superrosidae angiosperms and using critically evaluated fossil data for calibrating divergence-time estimates [35]. This was urgently needed because of conflicting hypotheses on Brassicaceae divergence-time estimates [36]. We built on this study [35] and expanded our sampling to 51 Brassicaceae plastomes and Cleome as outgroup. These species cover all four lineages or 5 out of 6 clades identified before $[5,17]$. We aimed to examine whether the plastome dataset could: (1) significantly support the previously shown deep splits; (2) resolve the disputed interrelationships between lineages or clades; and (3) reveal any previously overlooked structural evolution within Brassicaceae plastomes.

\section{Results}

Basic characteristics of Brassicaceae chloroplast genomes The average length of the plastomes from 53 species of Brassicales (Additional file 1: Table S1) is $154 \mathrm{~kb}$, ranging from 152,659 bp in Lobularia maritima to $160,100 \mathrm{bp}$ in Carica papaya (Additional file 1: Table S2). The average GC content is 36.4, 34.1, 29.3 and $42.3 \%$ for complete sequences, LSC, SSC and IR regions a and b, respectively, and varies slightly between species (Additional file 1: Table S2). As in the vast majority of angiosperms, both gene content and gene order are highly conserved, where the typical quadripartite organization harbored 132 genes including 79 protein coding genes (PCGs, 8 duplicated in IR), 30 tRNA genes (7 duplicated in IR) and 4 rRNA genes (4 duplicated in IR).

\section{Sequence alignment and evaluation of data partitions}

Based on the 77 PCGs, a gap-free supermatrix containing 64,962 sites was concatenated, of which 7611 were parsimony informative (Additional file 1: Table S3). The aligned lengths of these PCGs ranged from 84 to $6645 \mathrm{bp}$ $($ mean $=844 \mathrm{bp})$. No significant compositional heterogeneity among sequences was detected for any genes among species (Additional file 1: Table S4). The combined 77gene data set displayed no apparent substitution saturation (Additional file 1: Table S4). Evaluation of partition strategies suggested that the automatically determined scheme is the best according to Bayesian information criterion (BIC) and the most parameter-rich gene-codon model is generally better than the less partitioned ones, while the codon-partitioned model was favored over the gene-partitioned model (Additional file 1: Table S5).

\section{Plastome phylogeny}

Both RAxML and BEAST analyses of the concatenated sequence supermatrix produced similar topologies for the 53 species. For ML analysis, the topology and support values for specific splits varied using different partition schemes or subsets. After a visual check, no wellsupported conflicts (i.e., those receiving bootstrap (BS) $>90 \%$ ) were found between individual genes trees. Regardless of the data partition strategy in our ML analysis, the majority of relationships across the family were consistent and well supported. The BEAST topology based on the best-partition scheme defined by partition finder produced well-resolved phylogeny for all but two nodes (Fig. 1). In line with a recent conclusion [17], the three previously proposed lineages are placed into different clades. The placement of tribe Lepidieae was unstable across the analysis, and the alternative topology could not be rejected by approximately unbiased (AU) test $(P=0.297$, Table 1$)$. However, the relationship between clades determined by plastomes is discordant with nuclear gene phylogeny [17]. Of particular interest is the recently defined Clade E, a lineage containing a majority of Lineage III species, which is sister to the combined $\mathrm{BC}$ clade. Thus, after the split with basal Aethionemeae species, the core Brassicaceae diverged into two large clades. The first clade included species from Lineage I (or Clade A), while the second clade consisted of species from all other major lineages or clades except for the newly identified Clade D because of the limited taxon sampling. In addition, we found that 


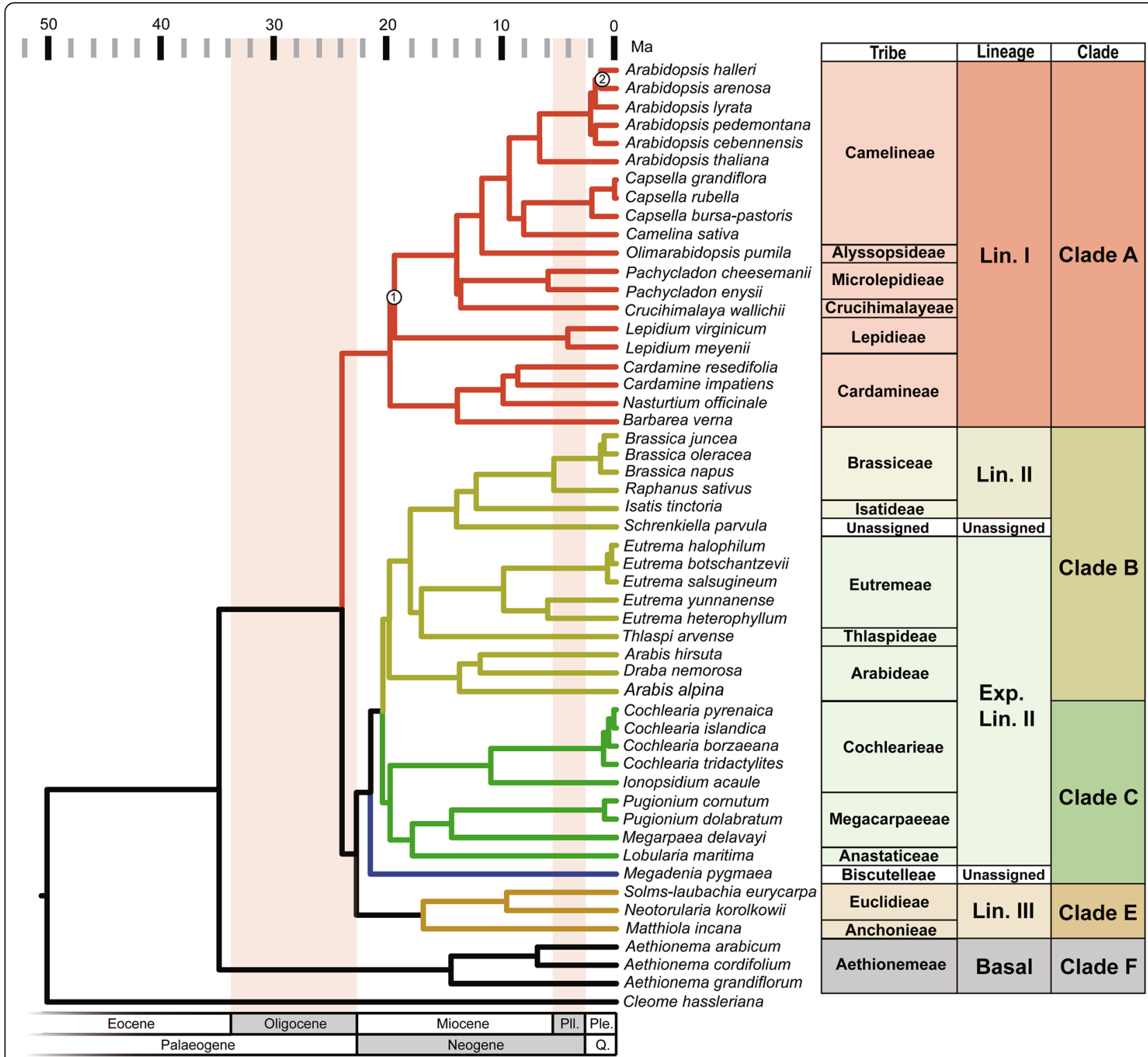

Fig. 1 Phylogeny of Brassicaceae. Time-calibrated phylogeny of 51 Brassicaceae species inferred from a concatenated Bayesian analysis of 77 plastome protein-coding genes with all mutations using BEAST2. Higher taxon names appear at right. All nodes are supported with posterior probability (PP) of 1.0, except for two marked with circles (Node1: PP = 0.558; Node2: PP = 0.833). Geological periods were marked with background colors. Ma million years ago, Ple Pleistocene, Pli Pliocene, Q. Quaternary

Table 1 Comparison of tree topology hypotheses by using likelihood

\begin{tabular}{lclllllll}
\hline Hypothesis & $\Delta \mathrm{InL}$ & $\mathrm{AU}$ & $\mathrm{BP}$ & $\mathrm{PP}$ & $\mathrm{KH}$ & WKH & $\mathrm{SH}$ \\
\hline $\mathrm{H} 1$ & Best & $\mathbf{0 . 7 5 6}$ & $\mathbf{0 . 7 2 1}$ & $\mathbf{0 . 9 7 7}$ & $\mathbf{0 . 7 3}$ & $\mathbf{0 . 7 3}$ & $\mathbf{0 . 9 6 6}$ & $\mathbf{0 . 9 8 6}$ \\
$\mathrm{H} 2$ & 3.7 & $\mathbf{0 . 2 9 7}$ & $\mathbf{0 . 2 7 3}$ & 0.023 & $\mathbf{0 . 2 7}$ & $\mathbf{0 . 2 7}$ & $\mathbf{0 . 6 9 3}$ & $\mathbf{0 . 5 5 5}$ \\
$\mathrm{H} 3$ & 25.3 & 0.011 & 0.006 & $1.00 \mathrm{E}-11$ & 0.014 & 0.014 & $\mathbf{0 . 1 7 6}$ & 0.026 \\
$\mathrm{H} 4$ & 27.9 & 0.003 & $4.00 \mathrm{E}-05$ & $7.00 \mathrm{E}-13$ & 0.007 & 0.007 & $\mathbf{0 . 1 4 5}$ & 0.022 \\
$\mathrm{H} 5$ & 118.1 & $8.00 \mathrm{E}-65$ & 0 & $5.00 \mathrm{E}-52$ & 0 & 0 & 0 \\
\hline
\end{tabular}

Note: Tree Hypothesis: H1. This study; H2. (Other CladeA + Cardamineae) + Lepidieae; H3. (((A, E), (B, C)), F); H4. (((A, (B, C)), E), F); H5. Megadenia within Clade C. $\triangle \mathrm{InL}$ : the observed log-likelihood difference. $A U$ approximately unbiased test, $B P$ bootstrap probability test, $P P$ approximate Bayesian posterior probability, $K H$ Kishino-Hasegawa test, WKH weighted Kishino-Hasegawa test, $S H$ Shimodaira-Hasegawa test, WSH weighted Shimodaira-Hasegawa test. $P$ values $>0.05$ are in bold. The topology for each alternative hypothesis is provided in Additional file 2: Figure S1 
Megadenia, a genus placed in the tribe Biscutelleae of Clade C, is sister to all other species of Clades B and C. Multiple tests confirmed the relationship recovered here and rejected the alternative phylogeny as previously proposed [17] $(P<0.01$, Table 1$)$.

\section{Fossil calibration and molecular dating}

We included plastomes of 75 outgroups in order to allow the use of 14 non-Brassicales calibrations (Additional file 2: Figure S2; Additional file 1: Table S6). A clock rate partition test found two partitions for the whole alignment as the best fit scheme under relaxed lognormal clock model. Overall, the calculation of divergence times were barely affected by whether fossil calibrations within the Brassicales were used (Table 2; Additional file 1: Table S7). Also, there was no effect on age estimation whether we included the questionable Thlaspi primaevum fossil [37] (here used as a conservative constrain to the Brassicaceae crown node as suggested [36]) or used the newly identified Palaeocleome lakensis fossil in the analysis [33] (Additional file 1: Table S7). According to the MCMCTREE time estimates, the core Brassicaceae and Aethionemeae began to split at 35.2 (30.0-42.5) Mya during the EoceneOligocene boundary (Fig. 1) while the origins of the major lineages or clades occurred between the late Oligocene and early Miocene (Table 2). These time estimates are broadly consistent with recent studies using

Table 2 Mean and 95\% HPD Age Estimates from MCMCTree Analysis

\begin{tabular}{lll}
\hline Node & \multicolumn{2}{l}{ Brassicales Fossils } \\
\cline { 2 - 3 } & Used & Not Used \\
\hline Cleomaceae vs Brassicaceae & $44.5-\mathbf{5 0 . 5}-59.1$ & $39.5-\mathbf{4 9 . 0}-57.6$ \\
Crown Brassicaceae & $30.0-\mathbf{3 5 . 2}-42.5$ & $29.0-\mathbf{3 4 . 9}-41.8$ \\
Crown core-Brassicaceae & $21.7-\mathbf{2 5 . 3}-29.7$ & $21.3-\mathbf{2 5 . 1}-29.8$ \\
Crown Clade A & $16.9-\mathbf{2 0 . 3}-24.2$ & $16.5-\mathbf{2 0 . 0}-24.1$ \\
Crown Arabidopsis & $4.8-\mathbf{7 . 1}-9.8$ & $4.8-\mathbf{7 . 0}-9.7$ \\
Crown Camelieae & $7.5-\mathbf{9 . 9}-12.8$ & $7.4-\mathbf{9 . 7}-12.5$ \\
Crown Cardamineae & $10.2-\mathbf{1 4 . 2}-18.6$ & $10.0-\mathbf{1 4 . 0}-18.3$ \\
Crown Clade B & $17.6-\mathbf{2 0 . 6}-24.5$ & $17.2-\mathbf{2 0 . 3}-24.4$ \\
Brassica vs Schrenkiella & $11.4-\mathbf{1 4 . 7}-18.3$ & $11.2-\mathbf{1 4 . 5}-18.2$ \\
Crown Eutremeae & $6.5-\mathbf{1 0 . 1}-14.3$ & $7.4-\mathbf{1 0 . 0}-14.2$ \\
Crown Arabideae & $11.0-\mathbf{1 4 . 6}-18.6$ & $10.8-\mathbf{1 4 . 4}-18.5$ \\
Crown Clade C & $16.9-\mathbf{2 0 . 1}-24.0$ & $16.6-\mathbf{1 9 . 8}-24.0$ \\
Crown Cochlearieae & $7.8-\mathbf{1 1 . 6}-15.9$ & $7.6-\mathbf{1 1 . 5}-15.9$ \\
Crown Megacarpaeeae & $10.1-\mathbf{1 4 . 8}-19.6$ & $10.0-\mathbf{1 4 . 6}-19.3$ \\
Megadenia vs BC clades & $19.3-\mathbf{2 2 . 6}-26.7$ & $18.3-\mathbf{2 2 . 3}-26.7$ \\
Crown Clade E & $12.7-\mathbf{1 7 . 3}-22.0$ & $12.4-\mathbf{1 7 . 0}-21.8$ \\
Crown Clade F & $9.5-\mathbf{1 4 . 6}-20.7$ & $9.3-\mathbf{1 4 . 3}-20.3$ \\
\hline
\end{tabular}

The numbers in boldface are mean values large-scale genomic data [5, 17, 34, 35]. Remarkably, all major lineages or clades radiated within a short timescales window ( 3 Myr between 17 and 20 Mya in the crown age; Fig. 1 and Table 2).

\section{Gene loss across Brassicaceae}

As shown in Fig. 2, of the total 79 PCGs, 77 were predicted to be functional genes while rps16 and $y c f 15$ became pseudogenes in some species (see also Additional file 2: Figures S3 and S4). Besides, the rpl22 gene was slightly truncated in Matthiola incana. The only exception was found for Solms-laubachia eurycarpa, where 10 of the $11 n d h$ genes were either slightly or severely truncated due to premature stop codons.

\section{Discussion}

In order to generate a backbone plastome phylogeny for Brassicaceae, we assembled 20 new plastomes to encompass all four lineages and 5 out of 6 major clades. All assumed lineages and major clades were generally identified, and their phylogenetic relationships were well resolved. In particular, our plastome phylogeny from 51 species provided the following new insights compared to those based on the fewer species plastome [35] or transcriptome genes [17]. First, Clade E, a group of Lineage III, is sister only to Clades $\mathrm{B}+\mathrm{C}$ instead of sister to Clades $\mathrm{B}+\mathrm{C}+\mathrm{A}$ [17]. Clade $\mathrm{A}$ diverged from the group comprised Clades $B+C+E$ very early. Second, the genus Megadenia of the tribe Biscutelleae in Clade $\mathrm{C}$ is sister to the remaining examined species of Clades B and C. This genus was shown to be closely related to Biscutella within the tribe Biscutelleae [38-41]. Thus, the phylogenetic relationship of the genus needs further examinations when more genera are sampled. Third, our comparisons based on different datasets suggested that the saturation in the third codon and phylogenetic signals from distinct plastome regions seriously affected the divergence and statistical supports in some particular nodes. For example, the tribes Lepidieae and others of Lineage I (Clade A) showed no distinct bifurcating divergence if all mutations of the total plastome dataset were used (Fig. 1). However, when excluding the third codon or using only slow-evolving IR genes, the Lepidieae diverged from Cardamineae and the others of the Lineage I (Clade A) with high statistical support (Additional file 2: Figures S5 and S6). Pachycladon was suggested to be closely related to Crucihimalaya $[37,42]$ as confirmed here by all plastome mutations (Fig. 1). However, this sister relationship was not supported when the IR gene dataset was used alone (Additional file 2: Figure S6).

Taxon sampling and reliable fossils used for calibrations are extremely important to estimate the divergence of targeted phylogeny [43-45]. Due to the high conservation and stable alignments, we used 14 highly reliable 


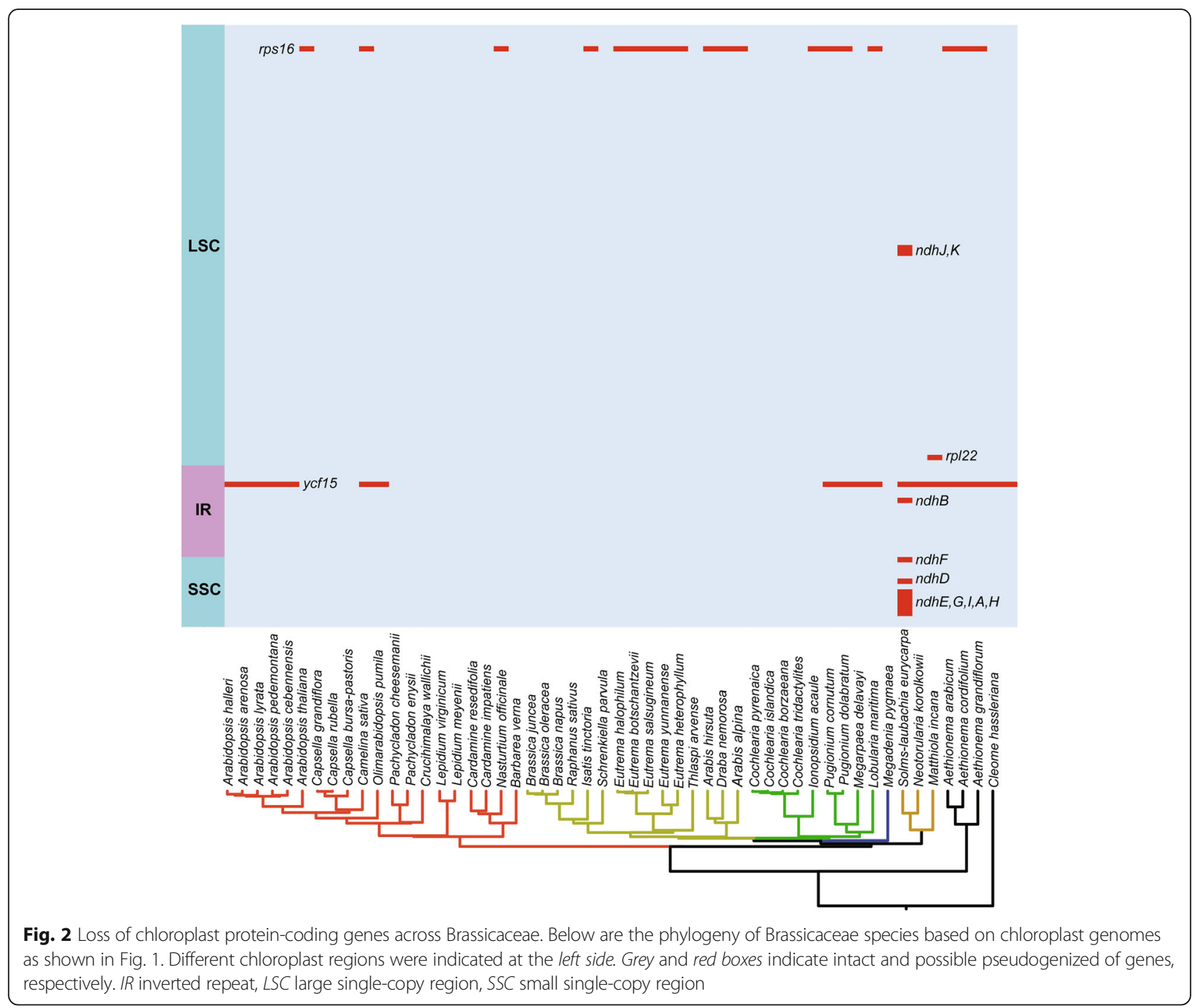

fossils from other eudicot orders [46] and three Brassicales. Our calibration comparisons suggested that the calculated divergences were rarely affected by Brassicales fossils, including the debated Brassicaceae fossil [37]. The estimated divergence times for major nodes were largely compatible with previous studies [5, 17, 34, 35], and it highlighted several evolutionary events. First, the stem age of Brassicaceae is around 50.5 Mya, 6 Mya older than estimated by Magallón et al. [46] and $\sim 5$ Mya younger than estimated by Huang et al. [17], but consistent with a recent study across the Brassicales order [33]. Second, we confirmed that Brassicaceae began to diversify $\sim 35.2$ Mya during the Eocene-Oligocene boundary [35], when a warm and humid climate dominated the world [47]. Third, all major clades or lineages radiated within a short timescale between $\sim 20$ and $\sim 17$ Mya. All of these estimates are non-significantly different from those based on the plastomes with fewer Brassicaceae species [35], but younger than those based on the low-copy nuclear genes [17] with more representatives at the genus level. Therefore, both taxon sampling and evolutionary rates of different genomes might have caused differences in the estimated node times between different datasets.

A few chloroplast genes were lost in photosynthetic plants [29]. In this study, we reaffirmed the loss of the rps16 gene in the LSC region [48] and found that the $y c f 15$ in the IR region became a pseudogene independently in different tribes of this family. Both genes were previously lost in other plants species [29]. The validity of $y c f 15$ as a protein-coding gene remains debated [49-51], and it may have a regulatory function [52] after the full transcription of the chloroplast genome [53]. Until now, the mechanism underlying the loss of the plastome gene in Brassicaceae has been poorly understood. The dominance of self-compatibility in the family might be related with the transfer and/or loss of some organelle genes $[48,54]$. However, it should be noted that Solms-laubachia eurycarpa has lost most ndh genes. To our 
knowledge, this is the first report of the massive loss of the $n d h$ genes in Brasssicales. A typical plastid genome contains $11 n d h$ genes that are highly conserved across most autotrophic seed plants, which indicates the presence of strong selection pressure for their retention. A complete loss of the plastid $n d h$ gene was only reported in conifers, Gnetales, and some epiphytic plants [55, 56]. Further studies are needed to examine whether specific factors were associated with the loss of the $n d h$ genes in the genus Solms-laubachia.

\section{Conclusions}

Recent emergence of large scale phylogenomic data have undoubtedly provided a major advancement for understanding the complex systematics and taxonomy of the Brassicaceae, while phylogenetic relationships of the entire large family is far from being fully resolved. Using 51 chloroplast genomes from species of major cruciferous lineages or clades, we were able to resolve deep splits in this important plant family and found incongruence between organelle and nuclear genomes. The updated phylogenetic framework, based on plastome analysis, can be used to test many interesting evolutionary hypothesis on the origin and early diversification of Brassicaceae species. With the rapid increase in genomic data, we envision that a further in-depth understanding of the evolution of this model plant family will soon be possible.

\section{Methods}

\section{Taxon sampling and plastome assembly}

A total of 53 Brassicales species were included in this study, among which were 51 Brassicaceae species from 28 genera representing 19 out of the 51 tribes in all four major lineages or 5 out of the 6 newly identified clades. Plastome sequences were either obtained from the NCBI (last accessed, Jan 1st, 2016) or newly assembled (Additional file 1: Table S1). For the newly sequenced plastomes, we used the Illumina HiSeq $\mathrm{X}$ Ten sequencing pipeline to generate at least $2 \mathrm{~Gb}$ of $2 \times 150$ bp short reads data for each sample. Reads from the SRA database were extracted with fastq-dump software implemented in the SRA toolkit. We initially filtered reads following the previous approach [57]. Then, plastome contigs were assembled using Velvet [58], which were further reordered to the Arabidopsis thaliana plastome with SAMtools [59]. We finally merged all contigs into a consensus linear sequence using Geneious version 8.0.5 [60]. The annotation was performed with CpGAVAS [61] or Plann [62], aided by manually refinement in Apollo genome editor [63] and/or Sequin software [64]. Aragorn web-interface [65] was used to predict tRNAs.

\section{Sequence alignment and partition strategy}

Protein coding genes (PCGs) were extracted from the Genbank formatted file containing all plastomes using customized Perl scripts, removing start and end codons. After excluding possible pseudogenes, a total of 77 PCGs were retained for all species except for Solms-laubachia eurycarpa, where the pseudogenized $n d h$ genes were edited and included. Each PCG was aligned using PRANK v.130410 [66] according to the translated amino acid sequences. Ambiguous alignment regions were trimmed by using Gblocks 0.91b [67] with $(-t=c)$ option to set sequence type to codons; otherwise the default settings were assumed.

To test the phylogenetic effects of different regions of the plastid genome, we created the following datasets based on different plastome partitions. All 77 refined PCG alignments were firstly combined into a concatenated data set and four different partitioning schemes: 1 partition (unpartitioned); 3 partitions (a separate partition for all first, second, and third codon positions); 77 partitions (one partition for each gene); and 231 partitions (a separate partition for the first and second codon positions together in each gene and a partition for the third codon position in each gene). In addition, a best-fit partitioning schemes and models were selected using the greedy search mode implemented in PartitionFinder v1.1.1 [68]. Comparisons of the five partitioning strategies and selections of corresponding nucleotide substitution models were conducted under the Bayesian information criterion (BIC). The best-fitting partitioning strategy found by PartitionFinder was used in the following analysis. In addition to the main dataset, we also extract subsets from the 77-gene alignments containing either first and second codon positions or third codon positions only to explore the effect of potential sequence saturation at third codon. The data matrices and resulting trees were deposited in TreeBase (http://purl.org/ phylo/treebase/phylows/study/TB2:S20512).

\section{Phylogenetic analysis}

The concatenated data set was first evaluated by $\mathrm{BaCoCa}$ [69], a recently developed program to perform multiple statistical analyses on multiple nucleotide and aminoacid sequence alignments, and then analyzed with Bayesian method and maximum likelihood (ML). The percentage of PI sites of each gene was estimated by PAUP [70]. The Bayesian MCMC analysis program BEAST (version 2.3.0) [71] was used to build phylogenetic trees, with parameter settings according to Hohmann et al. [35]. The GTR + G model was used for all ML analyses using RAxML version 8.0.20, as suggested in the manual [72]. Supports for nodes were assessed with 500 rapid bootstrapping replicates. Likelihood-based tests of alternative phylogenetic hypotheses were assessed based on the concatenated data 
set. Site-wise log-likelihoods of all alternative hypotheses (see Table 2) were first calculated with RAxML under the GTR + G model using the option ( $\mathrm{f} g$ ). Then, the site log-likelihood file was supplied to the CONSEL $\mathrm{v} 0.1 \mathrm{j}$ program [73] (Shimodaira and Hasegawa 2001) to estimate $P$-values for each alternative hypothesis using the AU test, approximate Bayesian posterior probability test, bootstrap probability test, Kishino-Hasegawa $(\mathrm{KH})$ test, weighted $\mathrm{KH}$ test, Shimodaira-Hasegawa test $(\mathrm{SH})$, and weighted $\mathrm{SH}$ test.

\section{Divergence-time estimation and fossil calibration}

We used the latest MCMCTREE in the PAML4.9a package to estimate divergence times with an approximate likelihood calculation [74], which allows a gamma-Dirichlet prior to describe substitution rates across multiple loci, thereby improving the accuracy of divergence-time estimation [75]. Optimal scheme for partitioning of the molecular clock(s) was tested for using ClockstaR 2.0.1 [76]. The ML phylogenetic tree topology from the 77 concatenated PCGs was used for divergence time estimation, and the ML branch lengths were estimated using the BASEML program in PAML under the GTR substitution model. For the gamma-Dirichlet prior for the overall substitution rate (rgene gamma), we used a quite diffuse (uninformative) prior $=1$. We used 14 highly reliable fossils from eudicot orders and three Brassicales fossils (Additional file 1: Table S6). All fossils were carefully selected according to their original descriptions and calibrations by past researches. Based on the mean estimate from three codon partitions using the strict molecular clock assuming $136 \mathrm{Ma}$ constraint at the root, an average of the eudicot-monocot split [46], the gamma-Dirichlet prior for the overall substitution rate (rgene gamma) was set at G $(4,80,1)$. The gamma-Dirichlet prior for the rate-drift parameter (sigma2 gamma) was set at $\mathrm{G}(1,10,1)$.

All calibration constraints were not rigorously constrained (specified with $2.5 \%$ tail probabilities above or below their limits; this is a built-in function of MCMCTREE). The independent rates model (clock $=2$ in MCMCTREE) [77] was used to specify the prior of rates among the internal nodes, which followed a log-normal distribution. The three parameters (birth rate $\lambda$, death rate $\mu$, and sampling fraction $\rho$ ) in the birth-death process with species sampling were specified as 1,1 , and 0 , respectively. After a burn-in period of 1,000,000 cycles, the MCMC run was sampled every 250 cycles until a total of 20,000 samples were collected. Two separate MCMC runs were compared for convergence with two different random seeds and similar results were observed. To explore the influence of our fossil calibrations on age estimates, we conducted four separate analyses testing the inclusion of various fossil combinations (Additional file 1: Table S7).

\section{Additional files}

\begin{abstract}
Additional file 1: Table S1. Species and data discription of cp genome used in this study. Table S2. Comparison of sequence length and GC content among Brassicales chloroplast genomes. Table S3. Comparison of fast-, intermediate-, and slow-evolving plastid protein-coding genes. Table S4. Heterogeneity and saturation test results from BaCoCa analysis. Table S5. Comparison of partitioning strategies. Table S6. Species and lineage specific fossil calibrations. Table S7. Comparison of mean and 95\% HPD age estimates using different fossils. (XLSX $48 \mathrm{~kb}$ )

Additional file 2: Figure S1. Topologies of alternative tree hypothesis used in approximately unbiased test. Figure S2. Chronogram of Brassicaceae and 75 outgroup taxa inferred using MCMCTree. Figure S3. Alignment view of Brassicales rps16 genes in MEGA6. Figure S4. Alignment view of Brassicales ycf15 genes in MEGA6. Figure S5. A phylogeny from ML analyses of 77 PCGs using 1st an 2nd codon. Figure S6. A phylogeny from ML analyses of 77 PCGs using genes from the IR region. Figure S7. A phylogeny from ML analyses of 77 PCGs using 3rd codon. Figure S8. A phylogeny from ML analyses of 77 PCGs using all three codons. (PDF $625 \mathrm{~kb}$ )
\end{abstract}

\section{Abbreviations}

AU: Approximately unbiased test; BIC: Bayesian information criterion; BP: Bootstrap; Bp: Base pair; GC: Guaninecytosine; IR: Inverted repeat region; LSC: Large single copy region; PCGs: Protein coding genes; SSC: Small single copy region

\section{Acknowledgements}

The authors would like to thank Qi He and Hao Bi for their technical help in the lab.

\section{Funding}

This work was supported by grants from the National Natural Science Foundation of China (31590821), and National Key Project for Basic Research (2014CB954100) and International Collaboration 111 Projects of China (2010DFA34610) from Ministry of Science and Technology of the People's Republic of China.

\section{Availability of data and materials}

Sequence data that support the findings of this study have been deposited in GenBank (accession numbers can be found in additional files). The phylogenetic matrix and trees are available in the TreeBASE repository (Study ID 20512).

\section{Authors' contributions}

$X G$ and $J L$ conceived the study and drafted the manuscript. XG performed the experiment, analyzed the data and interpreted the results. $\mathrm{GH}$ and $\mathrm{LZ}$ contributed plant material. KM and XW helped to analyze the data. DZ helped to perform the experiment and contributed the sequence data. KM, TM and QH contributed to the discussion of study design. IAA and MAK helped to improve the manuscript significantly. All authors read and approved the final manuscript.

\section{Competing interests}

The authors declare that they have no competing interests.

\section{Consent for publication \\ Not applicable.}

Ethics approval and consent to participate Not applicable.

\section{Author details}

${ }^{1}$ MOE Key Laboratory of Bio-Resources and Eco-Environment, College of Life Sciences, Sichuan University, 610065 Chengdu, People's Republic of China. ${ }^{2}$ Biodiversity Institute of Mount Emei, Mount Emei Scenic Area Management Committee, 614200 Leshan, Sichuan, People's Republic of China. ${ }^{3}$ Missouri Botanical Garden, PO Box 299, St. Louis, MO 63166-0299, USA. ${ }^{4}$ Department of Biodiversity and Plant Systematics, Im Neuenheimer Feld 345, Centre for Organismal Studies (COS) Heidelberg, Heidelberg University, 69120 Heidelberg, Germany. 


\section{Received: 23 October 2016 Accepted: 3 February 2017}

\section{Published online: 16 February 2017}

\section{References}

1. Al-Shehbaz IA, Beilstein MA, Kellogg EA. Systematics and phylogeny of the Brassicaceae (Cruciferae): an overview. Plant Syst Evol. 2006;259:89-120.

2. Bailey CD, Koch MA, Mayer M, Mummenhoff K, O'Kane SL, Warwick SI, et al. Toward a global phylogeny of the Brassicaceae. Mol Biol Evol. 2006;23:2142-60.

3. Koch MA, Dobeš C, Kiefer C, Schmickl R, Klimeš L, Lysak MA. Supernetwork identifies multiple events of plastid $\operatorname{trn} F(G A A)$ pseudogene evolution in the Brassicaceae. Mol Biol Evol. 2007;24:63-73.

4. Franzke A, German D, Al-Shehbaz IA, Mummenhoff K. Arabidopsis family ties: molecular phylogeny and age estimates in Brassicaceae. Taxon. 2009;58:425-37.

5. Couvreur TLP, Franzke A, Al-Shehbaz IA, Bakker FT, Koch MA, Mummenhoff K. Molecular phylogenetics, temporal diversification, and principles of evolution in the mustard family (Brassicaceae). Mol Biol Evol. 2010;27:55-71.

6. Novikova PY, Hohmann N, Nizhynska V, Tsuchimatsu T, Ali J, Muir G, et al. Sequencing of the genus Arabidopsis identifies a complex history of nonbifurcating speciation and abundant trans-specific polymorphism. Nat Genet. 2016;48:1077-82.

7. Lysak MA, Koch MA, Pecinka A, Schubert I. Chromosome triplication found across the tribe Brassiceae. Genome Res. 2005;15:516-25.

8. Mandakova T, Lysak MA. Chromosomal phylogeny and karyotype evolution in $x=7$ crucifer species (Brassicaceae). Plant Cell. 2008;20:2559-70.

9. Mandakova T, Joly S, Krzywinski M, Mummenhoff K, Lysak MA. Fast diploidization in close mesopolyploid relatives of Arabidopsis. Plant Cell. 2010;22:2277-90.

10. Edger PP, Heidel-Fischer HM, Bekaert M, Rota J, Glöckner G, Platts AE, et al. The butterfly plant arms-race escalated by gene and genome duplications. Proc Natl Acad Sci U S A. 2015;112:8362-6.

11. Al-Shehbaz IA. A generic and tribal synopsis of the Brassicaceae (Cruciferae) Taxon. 2012;61:931-54

12. Kiefer M, Schmickl R, German DA, Mandáková T, Lysak MA, Al-Shehbaz IA, et al. BrassiBase: introduction to a novel knowledge database on Brassicaceae evolution. Plant Cell Physiol. 2014:55:e3.

13. Al-Shehbaz IA, German DA, Mummenhoff K, Moazzeni H. Systematics, tribal placements, and synopses of the Malcolmia S.L. segregates (Brassicaceae). Harv Pap Bot. 2014;19:53-71.

14. German DA, Friesen NW. Shehbazia (Shehbazieae, Cruciferae), a new monotypic genus and tribe of hybrid origin from Tibet. Turczaninowia. 2014;17:17-23.

15. Beilstein MA, Al-Shehbaz IA, Kellogg EA. Brassicaceae phylogeny and trichome evolution. Am J Bot. 2006;93:607-19.

16. Lysak MA, Koch MA, Beaulieu JM, Meister A, Leitch IJ. The dynamic ups and downs of genome size evolution in Brassicaceae. Mol Biol Evol. 2009;26:85-98.

17. Huang $\mathrm{CH}$, Sun R, Hu Y, Zeng L, Zhang N, Cai L, et al. Resolution of Brassicaceae phylogeny using nuclear genes uncovers nested radiations and supports convergent morphological evolution. Mol Biol Evol. 2016;33:394-412.

18. Zeng L, Zhang Q, Sun R, Kong H, Zhang N, Ma H. Resolution of deep angiosperm phylogeny using conserved nuclear genes and estimates of early divergence times. Nat Commum. 2014;5:4956.

19. Zhang N, Zeng L, Shan H, Ma H. Highly conserved low-copy nuclear genes as effective markers for phylogenetic analyses in angiosperms. New Phytol. 2012;195:923-37.

20. Ruhfel BR, Gitzendanner MA, Soltis PS, Soltis DE, Burleigh JG. From algae to angiosperms-inferring the phylogeny of green plants (Viridiplantae) from 360 plastid genomes. BMC Evol Biol. 2014;14:1.

21. Ma PF, Zhang YX, Zeng CX, Guo ZH, Li DZ. Chloroplast phylogenomic analyses resolve deep-level relationships of an intractable bamboo tribe Arundinarieae (Poaceae). Syst Biol. 2014;63:933-50.

22. Carbonell-Caballero J, Alonso R, Ibañez V, Terol J, Talon M, Dopazo J. A phylogenetic analysis of 34 chloroplast genomes elucidates the relationships between wild and domestic species within the genus Citrus. Mol Biol Evol. 2015;32:2015-35.

23. Palmer JD. Comparative organization of chloroplast genomes. Annu Rev Genet. 1985;19:325-54.

24. Daniell H, Lin CS, Yu M, Chang WJ. Chloroplast genomes: diversity, evolution and applications in genetic engineering. Genome Biol. 2016;17:134.
25. Funk HT, Berg S, Krupinska K, Maier UG, Krause K. Complete DNA sequences of the plastid genomes of two parasitic flowering plant species, Cuscuta reflexa and Cuscuta gronovii. BMC Plant Biol. 2007;7:45.

26. McNeal JR, Kuehl JV, Boore $J \mathrm{~L}$, de Pamphilis $\mathrm{CW}$. Complete plastid genome sequences suggest strong selection for retention of photosynthetic genes in the parasitic plant genus Cuscuta. BMC Plant Biol. 2007:7:57.

27. Wolfe $\mathrm{KH}$, Morden CW, Palmer JD. Function and evolution of a minimal plastid genome from a nonphotosynthetic parasitic plant. Proc Natl Acad Sci U S A. 1992;89:10648-52.

28. Delannoy E, Fujii S, des Francs-Small CC, Brundrett M, Small I. Rampant gene loss in the underground orchid Rhizanthella gardneri highlights evolutionary constraints on plastid genomes. Mol Biol Evol. 2011;28:2077-86.

29. Magee AM, Aspinall S, Rice DW, Cusack BP, Sémon M, Perry AS, et al. Localized hypermutation and associated gene losses in legume chloroplast genomes. Genome Res. 2010;20:1700-10.

30. Jansen RK, Saski C, Lee SB, Hansen AK, Daniell H. Complete plastid genome sequences of three Rosids (Castanea, Prunus, Theobroma): evidence for at least two independent transfers of rpl22 to the nucleus. Mol Biol Evol. 2011; 28:835-47.

31. Ueda M, Fujimoto M, Arimura SI, Murata J, Tsutsumi N, Kadowaki KI. Loss of the $r p / 32$ gene from the chloroplast genome and subsequent acquisition of a preexisting transit peptide within the nuclear gene in Populus. Gene. 2007 402:51-6.

32. Jansen RK, Cai Z, Raubeson LA, Daniell H, Leebens-Mack J, Müller KF, et al. Analysis of 81 genes from 64 plastid genomes resolves relationships in angiosperms and identifies genome-scale evolutionary patterns. Proc Natl Acad Sci U S A. 2007;104:19369-74.

33. Cardinal-McTeague WM, Sytsma KJ, Hall JC. Biogeography and diversification of Brassicales: A 103million year tale. Mol Phylogenet Evol. 2016;99:204-24.

34. Kagale S, Robinson SJ, Nixon J, Xiao R, Huebert T, Condie J, et al. Polyploid evolution of the Brassicaceae during the Cenozoic era. Plant Cell. 2014;26:2777-91.

35. Hohmann N, Wolf EM, Lysak MA, Koch MA. A time-calibrated road map of Brassicaceae species radiation and evolutionary history. Plant Cell. 2015; 27(10):2770-84. doi:10.1105/tpc.15.00482.

36. Franzke A, Koch MA, Mummenhoff K. Turnip time travels: age estimates in Brassicaceae. Trends Plant Sci. 2016. doi:10.1016/j.tplants.2016.01.024.

37. Beilstein MA, Nagalingum NS, Clements MD, Manchester SR, Mathews S. Dated molecular phylogenies indicate a Miocene origin for Arabidopsis thaliana. Proc Natl Acad Sci U S A. 2010;107:18724-8.

38. German DA, Al-Shehbaz IA. Five additional tribes (Aphragmeae, Biscutelleae, Calepineae, Conringieae, and Erysimeae) in the Brassicaceae (Cruciferae). Harvard Pap Bot. 2008;13:165-70.

39. German DA, Friesen N, Neuffer B, Al-Shehbaz IA, Hurka H. Contribution to ITS phylogeny of the Brassicaceae, with a special reference to some Asian taxa. Plant Syst Evol. 2009;283:33-56.

40. Warwick SI, Mummenhoff K, Sauder CA, Koch MA, Al-Shehbaz IA. Closing the gaps: phylogenetic relationships in the Brassicaceae based on DNA sequence data of nuclear ribosomal ITS region. Plant Syst Evol. 2010;285:209-32.

41. Artyukova EV, Kozyrenko MM, Boltenkov EV, Gorovoy PG. One or three species in Megadenia (Brassicaceae): insight from molecular studies. Genetica. 2014;142:337-50.

42. Zhao B, Liu L, Tan D, Wang J. Analysis of phylogenetic relationships of Brassicaceae species based on Chs sequences. Biochem Syst Ecol. 2010;38:731-9.

43. Linder HP, Hardy CR, Rutschmann F. Taxon sampling effects in molecular clock dating: an example from the African Restionaceae. Mol Phylogenet Evol. 2005:35:569-82.

44. Mao K, Milne Rl, Zhang L, Peng Y, Liu J, Thomas P, et al. Distribution of living Cupressaceae reflects the breakup of Pangea. Proc Natl Acad Sci U S A. 2012:109:7793-8.

45. Mao KS, Liu JQ. Current 'relicts' more dynamic in history than previously thought. New Phytol. 2012;196:329-31.

46. Magallón S, Gómez-Acevedo S, Sánchez-Reyes LL, Hernández-Hernández T. A metacalibrated time-tree documents the early rise of flowering plant phylogenetic diversity. New Phytol. 2015;207:437-53.

47. Zachos J, Pagani M, Sloan L, Thomas E, Billups K. Trends, rhythms, and aberrations in global climate $65 \mathrm{Ma}$ to present. Science. 2001;292:686-93.

48. Roy S, Ueda M, Kadowaki Kl, Tsutsumi N. Different status of the gene for ribosomal protein S16 in the chloroplast genome during evolution of the genus Arabidopsis and closely related species. Genes Genet Syst. 2010;85:319-26 
49. Goremykin V, Hirsch-Ernst KI, Wölfl S, Hellwig FH. The chloroplast genome of the "basal" angiosperm Calycanthus fertilis -structural and phylogenetic analyses. Plant Syst Evol. 2003;242:119-35.

50. Raubeson LA, Peery R, Chumley TW, Dziubek C, Fourcade HM, Boore JL, Jansen RK. Comparative chloroplast genomics: analyses including new sequences from the angiosperms Nuphar advena and Ranunculus macranthus. BMC Genomics. 2007;8:174.

51. Tangphatsornruang S, Uthaipaisanwong P, Sangsrakru D, Chanprasert J, Yoocha T, Jomchai N, Tragoonrung S. Characterization of the complete chloroplast genome of Hevea brasiliensis reveals genome rearrangement, RNA editing sites and phylogenetic relationships. Gene. 2011;475:104-12.

52. Schmitz-Linneweber C, Maier RM, Alcaraz JP, Cottet A, Herrmann RG, Mache R. The plastid chromosome of spinach (Spinacia oleracea): complete nucleotide sequence and gene organization. Plant Mol Biol. 2001;45:307-15.

53. Shi C, Wang S, Xia EH, Jiang JJ, Zeng FC, Gao LZ. Full transcription of the chloroplast genome in photosynthetic eukaryotes. Sci Rep. 2016;6:30135. doi:10.1038/srep30135.

54. Andersson SG, Kurland CG. Reductive evolution of resident genomes. Trends Microbiol. 1998:6:263-8.

55. Martín M, Sabater B. Plastid ndh genes in plant evolution. Plant Physiol Biochem. 2010;48:636-45.

56. Blazier JC, Guisinger MM, Jansen RK. Recent loss of plastid-encoded ndh genes within Erodium (Geraniaceae). Plant Mol Biol. 2011;76:263-72.

57. Guo X, Hao G, Ma T. The complete chloroplast genome of salt cress (Eutrema salsugineum). Mitochondrial DNA A DNA Mapp Seq Anal. 2016; 27(4):2862-3. doi:10.3109/19401736.2015.1053130.

58. Zerbino DR, Birney E. Velvet: algorithms for de novo short read assembly using de Bruijn graphs. Genome Res. 2008;18:821-9.

59. Li H, Handsaker B, Wysoker A, Fennell T, Ruan J, Homer N, et al. The sequence alignment/map format and SAMtools. Bioinformatics. 2009;25:2078-9.

60. Kearse M, Moir R, Wilson A, Stones-Havas S, Cheung M, Sturrock S, et al. Geneious Basic: an integrated and extendable desktop software platform for the organization and analysis of sequence data. Bioinformatics. 2012;28:1647-9.

61. Liu C, Shi L, Zhu Y, Chen H, Zhang J, Lin X, Guan X. CpGAVAS, an integrated web server for the annotation, visualization, analysis, and GenBank submission of completely sequenced chloroplast genome sequences. BMC Genomics. 2012;13:715

62. Huang DI, Cronk QC. Plann: a command-line application for annotating plastome sequences. Appl Plant Sci. 2015;3(8). doi: 10.3732/apps.1500026.

63. Lewis SE, Searle SMJ, Harris N, Gibson M, lyer V, Richter J, et al. Apollo: a sequence annotation editor. Genome Biol. 2002;3:1.

64. Clark K, Karsch-Mizrachi I, Lipman DJ, Ostell J, Sayers EW. GenBank. Nucleic Acids Res. 2016;44(D1):D67-72. doi:10.1093/nar/gkv1276.

65. Laslett D, Canback B. ARAGORN, a program to detect tRNA genes and tmRNA genes in nucleotide sequences. Nucleic Acids Res. 2004;32:11-6.

66. Löytynoja A, Goldman N. Phylogeny-aware gap placement prevents errors in sequence alignment and evolutionary analysis. Science. 2008;320:1632-5.

67. Castresana J. Selection of conserved blocks from multiple alignments for their use in phylogenetic analysis. Mol Biol Evol. 2000;17:540-52.

68. Lanfear R, Calcott B, Ho SY, Guindon S. PartitionFinder: combined selection of partitioning schemes and substitution models for phylogenetic analyses. Mol Biol Evol. 2012;29:1695-701.

69. Kück P, Struck TH. BaCoCa-A heuristic software tool for the parallel assessment of sequence biases in hundreds of gene and taxon partitions. Mol Phylogenet Evol. 2014;70:94-8.

70. Swofford DL. PAUP*. Phylogenetic Analysis Using Parsimony (* and Other Methods). Version 4. MA: Sinauer Associates of Sunderland; 2003.

71. Bouckaert R, Heled J, Kühnert D, Vaughan T, Wu CH, Xie D, et al. BEAST 2: a software platform for Bayesian evolutionary analysis. PLoS Comput Biol. 2014;10:e1003537.

72. Stamatakis A. RAxML version 8: a tool for phylogenetic analysis and postanalysis of large phylogenies. Bioinformatics. 2014;30:1312-3.

73. Shimodaira H, Hasegawa M. CONSEL: for assessing the confidence of phylogenetic tree selection. Bioinformatics. 2001;17:1246-7.

74. Yang Z. PAML 4: phylogenetic analysis by maximum likelihood. Mol Biol Evol. 2007;24:1586-91.

75. dos Reis M, Zhu T, Yang Z. The impact of the rate prior on Bayesian estimation of divergence times with multiple loci. Syst Biol. 2014;63:555-65.

76. Duchêne S, Molak M, Ho SY. ClockstaR: choosing the number of relaxed-clock models in molecular phylogenetic analysis. Bioinformatics. 2014;30:1017-9.

77. Rannala B, Yang Z. Inferring speciation times under an episodic molecular clock. Syst Biol. 2007;56:453-66.

\section{Submit your next manuscript to BioMed Central and we will help you at every step:}

- We accept pre-submission inquiries

- Our selector tool helps you to find the most relevant journal

- We provide round the clock customer support

- Convenient online submission

- Thorough peer review

- Inclusion in PubMed and all major indexing services

- Maximum visibility for your research

Submit your manuscript at www.biomedcentral.com/submit 